\title{
A Novel Concatenated Code for Fading Channel
}

\author{
Ying Huang, Jing Lei, Baoguo Li, and Erbao Li
}

\begin{abstract}
A novel concatenated code is proposed for fading channel, which combines a linear outer code with a rate-1 inner code. Based on coding and diversity technique, higher reliability can be achieved without reducing efficiency because of inner code. In the paper, the rate- 1 inner code design is discussed in great detail. The design rule is shown as an optimized problem. According to different modulation and mapping style, the optimal inner coding matrixes are devised, which can be applied directly. Theoretical analysis shows that our proposed concatenated code obtains higher diversity order, which is adaptive for fading channel. Furthermore, it can be seen from the simulation result that the proposed concatenation can achieve better performance compared with the traditional concatenated code, even using the simpler outer code.
\end{abstract}

Index Terms-Concatenated code, fading channel, rate-1 inner code, multi-dimensional mapping.

\section{INTRODUCTION}

Under the fading channel, errors occur systematically because of path loss, which affects the information transmission heavily. Diversity techniques and channel codes are always used to enhance the reliability.

Concatenated codes, devised by Forney [1] in 1966, is a powerful technique for constructing long powerful codes from short component codes. In general, classical concatenation using a non-binary code as an outer code and a binary code as an inner code is widely used in both communication and digital data storage system to achieve high reliability with reduced decoding complexity. In most application, Reed-Solomon (RS) codes are used as the outer codes. Recently, other kinds of concatenation are devised, such as the concatenation with $\mathrm{LDPC}$ and $\mathrm{BCH}$ are used in DVB-S2 standard. Furthermore, Turbo codes [2], LDPC codes [3], IRA codes [4], fountain codes [5], polar codes [6] and quantum codes [7] can also be the component codes of concatenated scheme.

As we know, besides channel codes, diversity technique is another important method to debase error rate under the fading channel. The concatenated scheme based on channel codes and diversity technique is also preferred. Shan Ding etc [8] proposed a cascaded scheme which concatenated LDPC code with STBC code. Tarik Muharemovic et al. [9] considered the concatenation of channel code with space-time orthogonal transmit scheme. Geoffrey J. Byers et al. [10] have proposed a serially concatenated system employing an outer non-binary LDPC code and inner space-time trellis code.

Manuscript received January 5, 2015; revised June 25, 2015.

The authors are with Institute of Electronic Science and Engineering National University of Defense Technology, Changsha, China (e-mail: inform_huang@sina.com).
For the outstanding performance, it is worthy to discuss the combination of code and modulation. Multi-dimensional mapping technique [11]-[13], in which a group of binary bits is simultaneously mapped to multi-dimensional signal points, can enlarge the Euclidean distance. Furthermore, diversity order can be increased under the fading channel. In this paper, a novel concatenated code is proposed, which combines a linear outer code and a rate-1 inner code. Compared with others, the proposed concatenated scheme has two merits: 1) the inner code does not reduce the whole code rate; 2 ) the diversity order can be increased which profits from the advantage of multi-dimensional mapping.

The paper is organized as follows. In Section II, the novel concatenated code is proposed. We discuss the rate-1 inner code design in Section III. Some theoretical analysis and simulation result are presented in Section IV, and finally we provide some concluding remarks in the last section.

\section{The Novel Concatenated Code}

In general, a simple concatenated code (Fig. 1) is formed from two codes: an $\left(n_{1}, k_{1}\right)$ binary code $C_{1}$ and an $\left(n_{2}, k_{2}\right)$ non-binary code $C_{2}$ with symbols from $G F\left(2^{k_{1}}\right)$. Concatenated codes are effective against a mixture of random errors and bursts. Usually, an RS code is used for $C_{2}$, which is recommended in CCSDS, IEEE 802.11 et al.

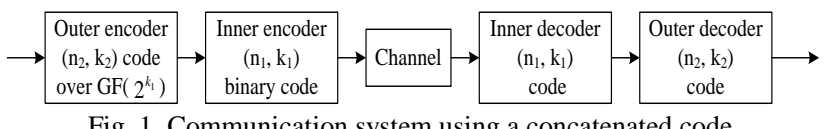

The communication system using the proposed concatenated code (in dashed square) is shown as Fig. 2. The outer code can be traditional linear block codes (BCH, RS et al.), convolutional codes (CC), turbo codes and LDPC codes. The inner code is a rate- 1 code, which is different from the traditional concatenation. That means, the whole rate of concatenation won't reduce because of inner code.

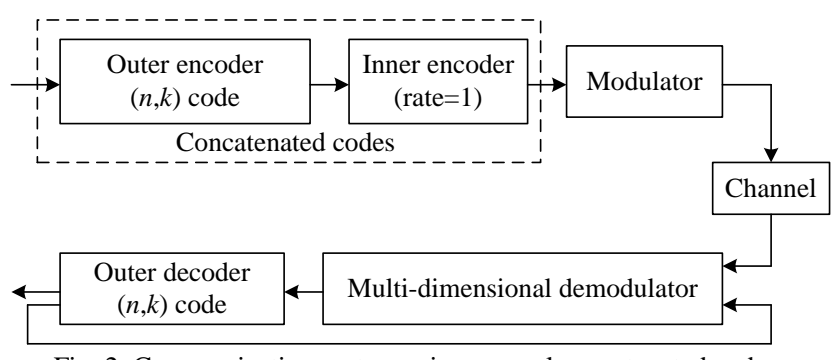

Fig. 2. Communication system using a novel concatenated code.

Whatever the channel code is used as outer code, we assume that the length of a code word (block codes) or code vector according to $k$-bit information vector (convolutional 
codes or turbo codes) is $n$. Furthermore, the order of modulation is $M=2^{m}$.

Encoding consists of two steps. First, the $k$ bits are encoded according to the rules for $(n, k)$ code to form an $n$-bit code word. Then, we divide $n$ bits into several $L m$-bit block ( $L \in Z^{+}$). For simplicity in discussion, it is assumed that $n$ can be divided by $L m$ exactly, i.e. $n=q L m$, $q \in Z^{+}$.The code word can be denoted as $\boldsymbol{C}=\left[\boldsymbol{c}_{1}, \boldsymbol{c}_{2}, \ldots, \boldsymbol{c}_{q}\right]^{T}$, and $\boldsymbol{c}_{i}=\left[c_{1}^{i}, c_{2}^{i}, \ldots c_{L m}^{i}\right]^{T}, c_{j}^{i} \in G F(2)$ is the $i$ th $L m$-bit block $(i=1,2, \ldots q ; j=1,2, \ldots, L m)$.

Second, each $L m$-bit block is encoded by rate-1 inner code, i.e. $\boldsymbol{v}_{i}=\boldsymbol{G} \cdot \boldsymbol{c}_{i} . \boldsymbol{G}$ is a $L m \times L m$ square matrix in $\mathrm{GF}(2)$. Then, each $L m$-bit block can be modulated as one multi-dimensional symbol $\boldsymbol{s}_{i}$, denoted as $\boldsymbol{s}_{i}=\left(s_{1}^{i}, s_{2}^{i}, \ldots, s_{L}^{i}\right), s_{j}^{i} \in \psi$, where $\psi$ is the constellation of $M$-ary modulation, and $s_{i}$ is a $L$-dimensional symbol. For the whole $n$-bit code word, the modulated symbol vector can be viewed as a multi-dimensional symbol vector, which is denoted as $\boldsymbol{S}=\left(\boldsymbol{s}_{1}, \boldsymbol{s}_{2}, \ldots, \boldsymbol{s}_{q}\right), \boldsymbol{s}_{i} \in \Psi$, and $\Psi=\psi^{\mathrm{L}}$.

The code rate of proposed concatenated codes is $k / n$, which only depends on the outer code. Compared with traditional concatenated code, the receiver of our scheme is evidently different. The received signal vector is demodulated first, which is based on multi-dimensional mapping technique. Then, LLR value for each bit is transmitted to outer decoder. The external information of decoder can be delivered back to demodulator for iteration. The whole communication system can be considered as a BICM-ID system.

\section{INNER CODES DESIGN}

For our proposed concatenated code, the inner code is special, which is different from the traditional concatenation. In this section, the design of inner codes will be discussed in detail.

\section{A. Design Rule}

For simplicity, $\boldsymbol{v}_{i}$ and $\boldsymbol{c}_{i}$ can be substituted by $\boldsymbol{v}$ and $\boldsymbol{c}$ respectively. Under the wireless fading channel, when design the inner code matrix $\boldsymbol{G}$, there are two items should be considered [13], [14]:

1). Maximizing the Euclidean distance between any two multi-dimensional symbol vectors, when only 1 bit is different in their mapping bit vectors;

2). Full rank.

Let $c_{1}$ and $c_{2}$ are any two bit vectors, the $i$ th bit is different. As follow:

$$
\boldsymbol{c}_{1} \oplus \boldsymbol{c}_{2}=\left[\begin{array}{lllllll}
0 & \cdots & 0 & 1 & 0 & \cdots & 0
\end{array}\right]^{T}
$$

After rate-1 inner code, $\boldsymbol{v}_{1}=\boldsymbol{G} \cdot \boldsymbol{c}_{1}, \boldsymbol{v}_{2}=\boldsymbol{G} \cdot \boldsymbol{c}_{2}$.

$$
v_{1} \oplus v_{2}=\boldsymbol{G} \cdot\left(c_{1} \oplus c_{2}\right)=g_{i}
$$

where " $\oplus$ " is addition over GF(2), $\boldsymbol{g}_{i}$ is the $i$ th column of $\boldsymbol{G}$.

Then, $\boldsymbol{v}_{1}$ and $\boldsymbol{v}_{2}$ are mapped to symbol vectors $\boldsymbol{s}_{1}=\left(s_{1}^{1}, s_{2}^{1}, \ldots, s_{L}^{1}\right) \quad$ and $\boldsymbol{s}_{2}=\left(s_{1}^{2}, s_{2}^{2}, \ldots, s_{L}^{2}\right) \quad$ respectively, which means $s_{1}$ is the multi-dimensional symbol to $c_{1}$, and $\boldsymbol{s}_{2}$ is the multi-dimensional symbol to $\boldsymbol{c}_{2}$. The Euclidean distance between those two multi-dimensional symbols is

$$
\left\|\boldsymbol{s}_{1}-\boldsymbol{s}_{2}\right\|^{2}=\sum_{i=1}^{L}\left\|s_{i}^{1}-s_{i}^{2}\right\|^{2}
$$

The design rule of optimal inner coding matrix $\boldsymbol{G}$ is written as follow:

$$
\begin{gathered}
\max _{G}\left\{\left\|s_{1}-s_{2}\right\|^{2}\right\} \\
\text { s.t. } w\left(\boldsymbol{c}_{1} \oplus \boldsymbol{c}_{2}\right)=1 \\
\boldsymbol{v}_{1}=\boldsymbol{G} \cdot \boldsymbol{c}_{1}, \quad \boldsymbol{v}_{2}=\boldsymbol{G} \cdot \boldsymbol{c}_{2} \\
\boldsymbol{s}_{1}=\xi_{\psi}\left(\boldsymbol{v}_{1}\right), \boldsymbol{s}_{1}=\xi_{\psi}\left(\boldsymbol{v}_{2}\right) \\
|\boldsymbol{G}| \neq 0
\end{gathered}
$$

where $w(\cdot)$ is hamming weight of vectors, and $\xi_{\psi}$ is mapping style in constellation $\psi$.

\section{B. BPSK/QPSK}

For BPSK, "1" is mapped to " +1 ","0" is mapped to "- 1 ". Based on Eq.(4), the optimal $\boldsymbol{G}$ can be written as

$$
\boldsymbol{G}=\left[\begin{array}{cccc}
1 & 1 & \cdots & 1 \\
1 & 0 & 1 & 1 \\
& \vdots & & \vdots \\
1 & 1 & \cdots & 0
\end{array}\right]_{L m \times L m}
$$

It can be seen from (5) that the optimal $\boldsymbol{G}$ matrix has the following traits:

1). Only one column has $L m$ entries of "1";

2). $L m-1$ columns comprise $L m-1$ entries of " 1 " and one entry of " 0 ".

For QPSK with Gray, the optimal $\boldsymbol{G}$ is the same as (5) [15]. For QPSK with anti-Gray, for any two bits $v_{1}, v_{2}$, the modulated symbol is $s=\left[2\left(v_{1} \oplus v_{2}\right)-1,2 v_{1}-1\right]$. Based on (4), the optimal $\boldsymbol{G}$ can be written as

$$
\boldsymbol{G}=\left[\begin{array}{ccccccccc}
1 & 1 & 1 & & 1 & 0 & 1 & & 1 \\
0 & 1 & 0 & & 0 & 1 & 0 & & 0 \\
1 & 1 & 1 & \cdots & 1 & 1 & 0 & \cdots & 1 \\
0 & 0 & 1 & & 0 & 0 & 1 & & 0 \\
& & \vdots & & & & \vdots & & \\
& & & & & & & & \\
1 & 1 & 1 & \cdots & 1 & 1 & 1 & \cdots & 1 \\
0 & 0 & 0 & & 1 & 0 & 0 & & 0
\end{array}\right]_{L m \times L m}
$$


For QPSK with anti-Gray, the optimal $\boldsymbol{G}$ matrix has the following traits:

1). Only one column has $L m / 2$ entries of $\left[\begin{array}{ll}1 & 0\end{array}\right]^{T}$;

2). $L m / 2$ columns comprise $L m / 2-1$ entries of $\left[\begin{array}{ll}1 & 0\end{array}\right]^{T}$ and one entry of $\left[\begin{array}{ll}1 & 1\end{array}\right]^{T}$;

3). $L m / 2-1$ columns comprise $L m / 2-1$ entries of $\left[\begin{array}{ll}1 & 0\end{array}\right]^{T}$ and one entry of $\left[\begin{array}{ll}0 & 1\end{array}\right]^{T}$.

The analogy result can also be found in Ref.[16].

\section{High-Order Modulation}

For high-order modulation, we have no straight way to obtain the optimal $\boldsymbol{G}$ of Eq.(4). That means, an indirect method is needed. First, we define the Euclidean distance matrix $\boldsymbol{E D}$ as Eq.(7).

$$
\boldsymbol{E D}=\left[\begin{array}{cccc}
\left|s_{1,1}^{1}-s_{1,1}^{2}\right|^{2} & \left|s_{1,2}^{1}-s_{1,2}^{2}\right|^{2} & \cdots & \left|s_{1, L m}^{1}-s_{1, L m}^{2}\right|^{2} \\
\left|s_{2,1}^{1}-s_{2,1}^{2}\right|^{2} & \left|s_{2,2}^{1}-s_{2,2}^{2}\right|^{2} & & \left|s_{2, L m}^{1}-s_{2, L m}^{2}\right|^{2} \\
\vdots & & \vdots \\
\left|s_{L-1,1}^{1}-s_{L-1,1}^{2}\right|^{2} & \left|s_{L-1,2}^{1}-s_{L-1,2}^{2}\right|^{2} & & \left|s_{L-1, L m}^{1}-s_{L-1, L m}^{2}\right|^{2} \\
\left|s_{L, 1}^{1}-s_{L, 1}^{2}\right|^{2} & \left|s_{L, 2}^{1}-s_{L, 2}^{2}\right|^{2} & \cdots & \left|s_{L, L m}^{1}-s_{L, L m}^{2}\right|^{2}
\end{array}\right]_{L \times L m}
$$

$\boldsymbol{E D}$ has $L$ rows and $L m$ columns. It is assumed that only $j$ th bit is different in $c_{j}^{1}$ and $c_{j}^{2}, j \in\{1,2, \ldots, L m\}$.The coded vector are $\quad \boldsymbol{v}_{j}^{1}=\left[v_{1, j}^{1}, v_{2, j}^{1}, \ldots, v_{L m, j}^{1}\right]^{T} \quad$ and $\boldsymbol{v}_{j}^{2}=\left[v_{1, j}^{2}, v_{2, j}^{2}, \ldots, v_{L m, j}^{2}\right]^{T}$, which satisfied $\boldsymbol{v}_{j}^{1}=\boldsymbol{G} \cdot \boldsymbol{c}_{j}^{1}$ and $\boldsymbol{v}_{j}^{2}=\boldsymbol{G} \cdot \boldsymbol{c}_{j}^{2}$.Then, $\boldsymbol{v}_{j}^{1}$ is mapped to $L$-dimensional symbol vector $\boldsymbol{s}_{j}^{1}=\left[s_{1, j}^{1}, s_{2, j}^{1}, \ldots, s_{L, j}^{1}\right]^{T}$, and $\boldsymbol{v}_{j}^{2}$ is mapped to $L$-dimensional symbol vector $s_{j}^{2}=\left[s_{1, j}^{2}, s_{2, j}^{2}, \ldots, s_{L, j}^{2}\right]^{T}$. The entry at $i$ th row and $j$ th column of $\boldsymbol{E D}$ is the Euclidean distance between the $i$ th symbol of $\boldsymbol{s}_{j}^{1}$ and $\boldsymbol{s}_{j}^{2}$.

For M-ary modulation, the equivalent design rule can be written as follow:

$$
\begin{gathered}
\max _{\boldsymbol{G}}\left\{\|\boldsymbol{E D}\|_{(1)}\right\} \\
\text { s.t. }|\boldsymbol{G}| \neq 0 \\
\boldsymbol{G}=\left[\boldsymbol{g}_{1}, \boldsymbol{g}_{2}, \ldots, \boldsymbol{g}_{L m}\right], \boldsymbol{g}_{j}=\left[g_{1}^{j}, g_{2}^{j}, \ldots, g_{L m}^{j}\right]^{T} \\
{\left[v_{m i-m+1, j}^{1} \oplus v_{m i-m+1, j}^{2}, v_{m i-m+2, j}^{1} \oplus v_{m i-m+2, j}^{2}, \ldots, v_{m i, j}^{1} \oplus v_{m i, j}^{2}\right]^{T}} \\
=\left[g_{m i-m+1}^{j}, g_{m i-m+2}^{j}, \ldots, g_{m i}^{j}\right]^{T} \\
\xi_{\psi}\left(v_{m i-m+1, j}^{1}, v_{m i-m+2, j}^{1}, \ldots, v_{m i, j}^{1}\right)=s_{i, j}^{1} \\
\xi_{\psi}\left(v_{m i-m+1, j}^{2}, v_{m i-m+2, j}^{2}, \ldots, v_{m i, j}^{2}\right)=s_{i, j}^{2} \\
i=1,2, \ldots, L, \quad j=1,2, \ldots, L m
\end{gathered}
$$

where $\|\boldsymbol{E} \boldsymbol{D}\|_{(1)}=\sum_{i=1}^{L} \sum_{j=1}^{L m}\left|s_{i, j}^{1}-s_{i, j}^{2}\right|^{2}$, and $\|\cdot\|_{(1)}$ is $p$ norm $(p=1)$.

For different high-order modulation, we should analyze the relationship between Euclidean distance of any two modulated symbols and the XORed result of their mapping bit vectors. That means, optimal $\boldsymbol{G}$ is different for different mapping style, even in the same modulation.

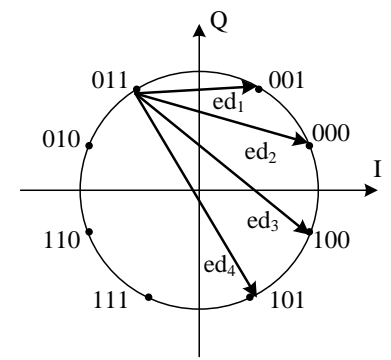

Fig. 3. 8PSK with gray.

We take 8PSK with Gray (Fig. 3) as an example to discuss the method how to obtain the optimal $\boldsymbol{G}$ based on (8). There are four kinds of Euclidean distance in constellation, such as $e d_{1}, e d_{2}, e d_{3}$ and $e d_{4}$. Under the design rule (8), $\boldsymbol{E D}$ matrix, which is according to optimal $\boldsymbol{G}$, can be denoted as follow:

$$
\boldsymbol{E D}=\left[\begin{array}{cccccccc}
e d_{4} & e d_{3} & e d_{4} & \cdots & e d_{4} & e d_{2} & \cdots & e d_{4} \\
e d_{4} & e d_{4} & e d_{3} & \cdots & e d_{4} & e d_{4} & \cdots & e d_{4} \\
& \vdots & & & & \vdots & & \\
e d_{4} & e d_{4} & & & e d_{4} & e d_{4} & \cdots & e d_{2} \\
e d_{4} & e d_{4} & e d_{4} & e d_{4} & e d_{3} & e d_{4} & \cdots & e d_{4}
\end{array}\right]_{L \times 3 L}
$$

From (9), the $\boldsymbol{E D}$ matrix has the following traits:

1). Only one column has $L$ entries of $e d_{4}$;

2). $L$ columns comprise $L-1$ entries of $e d_{4}$ and one entry of $\mathrm{ed}_{3}$;

3). $2 L-1$ columns comprise $L-1$ entries of $e d_{4}$ and one entry of $e d_{2}$.

When $L m=6, \boldsymbol{E D}$ can be written as

$$
\boldsymbol{E} \boldsymbol{D}=\left[\begin{array}{llllll}
e d_{4} & e d_{4} & e d_{3} & e d_{2} & e d_{4} & e d_{4} \\
e d_{4} & e d_{3} & e d_{4} & e d_{4} & e d_{2} & e d_{2}
\end{array}\right]
$$

In order to obtain the largest Euclidean distance $e d_{4}$, the XORed result of two mapping vectors must be $\left[\begin{array}{lll}1 & 1 & 0\end{array}\right]^{T}$. Similarly, $e d_{3}$ is corresponding to $\left[\begin{array}{lll}1 & 1 & 1\end{array}\right]^{T}$, and $e d_{2}$ is corresponding to $\left[\begin{array}{lll}1 & 0 & 1\end{array}\right]^{T}$ or $\left[\begin{array}{lll}0 & 1 & 1\end{array}\right]^{T}$. Then, the optimal $\boldsymbol{G}$ can be constructed based on the optimal $\boldsymbol{E D}$, which can be denoted as

$$
\boldsymbol{G}=\left[\begin{array}{llllll}
1 & 1 & 1 & 1 & 1 & 1 \\
1 & 1 & 1 & 0 & 1 & 1 \\
0 & 0 & 1 & 1 & 0 & 0 \\
1 & 1 & 1 & 1 & 1 & 0 \\
1 & 1 & 1 & 1 & 0 & 1 \\
0 & 1 & 0 & 0 & 1 & 1
\end{array}\right]
$$


In conclusion, the optimal $\boldsymbol{G}$ based on optimization problem (shown as (8)) can be achieved by three steps:

Step 1: find the optimal $\boldsymbol{E D}$ based on different modulation and different mapping style;

Step 2: find the relationship between Euclidean distance and the XORed result of two mapping vectors;

Step 3: deduce the optimal $\boldsymbol{G}$ from the optimal $\boldsymbol{E D}$.

The above method is general for other high-order modulation and different mapping style.

\section{THEORETICAL ANALYSIS AND SIMULATION}

\section{A. Performance Advantage for Fading Channel}

In this section, we will deduce the theoretical performance of the proposed concatenation, and discuss its advantage for fading channel. Without loss of generality, we assume $S$ and $\tilde{\boldsymbol{S}}$ differ in the first $d$ consecutive $L$-dimensional symbols, which can be defined as

$$
\begin{aligned}
& \boldsymbol{S}=\left(\boldsymbol{s}_{1}, \boldsymbol{s}_{2}, \ldots, \boldsymbol{s}_{d}\right)=\left(s_{1}^{1}, \ldots, s_{L}^{1}, s_{1}^{2}, \ldots, s_{L}^{2}, \ldots, s_{1}^{d}, \ldots, s_{L}^{d}\right) \\
& \tilde{\boldsymbol{S}}=\left(\tilde{\boldsymbol{s}}_{1}, \tilde{\boldsymbol{s}}_{2}, \ldots, \tilde{\boldsymbol{s}}_{d}\right)=\left(\tilde{s}_{1}^{1}, \ldots, \tilde{s}_{L}^{1}, \tilde{s}_{1}^{2}, \ldots, \tilde{s}_{L}^{2}, \ldots, \tilde{s}_{1}^{d}, \ldots, \tilde{s}_{L}^{d}\right)
\end{aligned}
$$

where $d$ is the hamming distance of the outer code, and $\boldsymbol{s}_{i}, \tilde{\boldsymbol{s}}_{i} \in \Psi, i=1,2, \ldots, d$.

Given coherent detection and maximum likelihood decoding, the pairwise error probability (PEP) [14] conditioned on $\boldsymbol{H}$ can be computed as

$$
P(d \mid \boldsymbol{H})=Q\left(\sqrt{\frac{1}{2 \sigma^{2}} \sum_{i=1}^{d} \sum_{j=1}^{L}\left|h_{j}^{i}\right|^{2} \cdot P \cdot\left|s_{j}^{i}-\tilde{s}_{j}^{i}\right|^{2}}\right)
$$

where $P$ is the transmitted power, and $\sigma^{2}$ is the noise variance. $\boldsymbol{H}=\left(\boldsymbol{h}_{1}, \boldsymbol{h}_{2}, \ldots, \boldsymbol{h}_{d}\right)$ is the channel coefficient, and $\boldsymbol{h}_{i}=\left(h_{1}^{i}, h_{2}^{i}, \ldots, h_{L}^{i}\right), E\left[\left|u_{i}\right|^{2}\right]=1, E\left[\left\|\boldsymbol{s}_{i}\right\|^{2}\right]=L, i=1,2, \ldots, d$.

Based on Eq.(12), after average in whole constellation, then the PEP can be calculated as follow:

$$
\begin{aligned}
& f\left(d, \Psi, \xi_{\Psi}\right) \leq \frac{1}{2} \cdot E\left\{\prod_{p=1}^{L}\left(1+P \cdot \frac{\left|s_{p}-s_{p}^{(k)}\right|^{2}}{4 \sigma^{2}}\right)^{-1}\right\}^{d} \\
& =\frac{1}{2} \cdot\left\{\frac{1}{m L \cdot 2^{m L}} \sum_{s \in \Psi} \sum_{k=1}^{m L}\left[\prod_{p=1}^{L}\left(1+P \cdot \frac{\left|s_{p}-s_{p}^{(k)}\right|^{2}}{4 \sigma^{2}}\right)^{-1}\right]\right\}^{d}
\end{aligned}
$$

where $\boldsymbol{s}_{(k)}, \boldsymbol{s}$ are the $L$-dimensional symbols in constellation $\Psi$, and only $k$ th bit is different between their mapping bit vectors, $\boldsymbol{s}_{p} \in \boldsymbol{S}, \boldsymbol{s}_{p}^{(k)} \in \boldsymbol{S}_{(k)}$.

From (13), when $s_{p} \neq s_{p}^{(k)}(p=1, \ldots, L), L \cdot d$-order diversity can be achieved with the help of the rate- 1 inner code. That means, on the premise of the fixed outer code and modulation order, the diversity order is increasing as $L$ increasing, which is adaptive for fading channel.

\section{B. Simulation and Analysis}

In simulation, the traditional concatenated code combines $\operatorname{RS}(38,34)$ in $\operatorname{GF}\left(2^{6}\right)$ with $\mathrm{CC}(2,1,7)$, whose generator polynomial is [133 171]. Otherwise, $\mathrm{CC}(2,1,3)$ and rate-1 code( $L=3, m=2$ ) are cascaded as the proposed concatenation. In traditional scheme, the length of code block is 456 , and the whole code rate is 0.45 . Analogously, the length of code block is 512, and whole code rate is 0.5 in our proposed concatenation. Otherwise, QPSK and fading channel are used in simulation.

For the proposed scheme, Max-log-MAP algorithm and log-MAP algorithm are used in multi-dimensional demodulated and decoding respectively. The number of iteration is 8 . In traditional scheme, hard-decision decoding is used in both outer decoder and inner decoder.

Fig. 4 shows the performance comparison between the traditional concatenation and the proposed one. From the figure, we can see that the proposed concatenated code outperform the traditional one (RS+CC). At BER $=10^{-5}, 1.5 \mathrm{~dB}$ gain can be achieved by proposed scheme. On the premise of simple code $(\mathrm{CC}(2,1,3))$, the performance advantage is derived from two aspects: 1) the diversity predominance because of rate-1 inner code; 2) iterative decoding between demodulator and decoder at receiver, which is based on soft information.

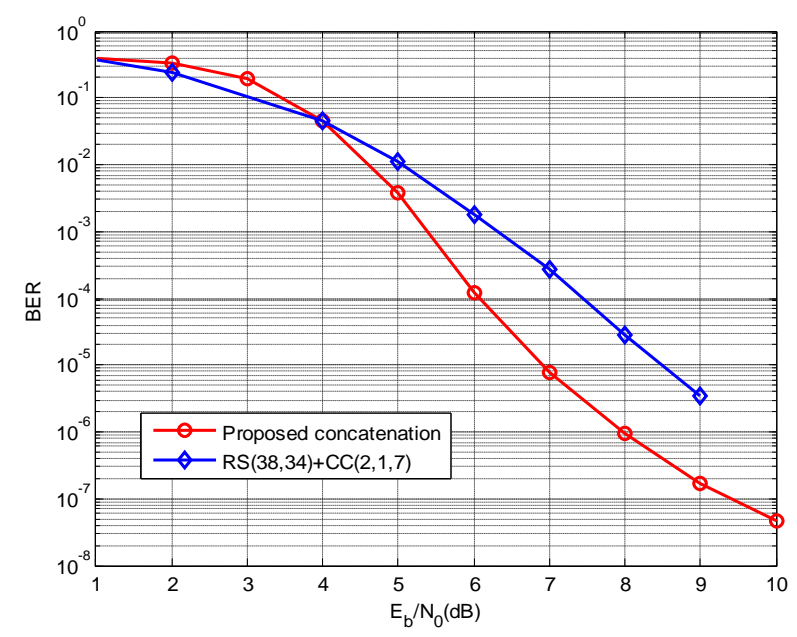

Fig. 4. Performance comparison.

\section{CONCLUSION}

Under the fading channel, a novel concatenated code is proposed, which combines an outer code with a rate- 1 inner code. The design of inner code is discussed in detail. The universal design rule is shown as optimization problem, and the optimal encoding matrixes are deduced for different modulation and mapping style. Both theoretical performance analysis and simulation result all show that our proposed concatenation can achieve better performance, even a simpler outer code is used.

\section{ACKNOWLEDGMENT}

The authors would like to thank the reviewers for their 
detailed reviews and constructive comments, which have helped improve the quality of this paper. This work was supported in part by a grant from the Ph.D. Programs Foundation of Ministry of Education of China under Grants No. 20094307110004, National Natural Science Foundation of China under Grants No.61372098, No.61101074 and Natural Science Foundation of Hunan Province, China under Grants No.12jj2037.

\section{REFERENCES}

[1] G. D. Forney, Concatenated Codes, Cambridge: MIT Press, 1966

[2] A. Perotti, G. Montorsi, and S. Benedetto, "Performance analysis and optimization of concatenated block-turbo coding schemes," IEEE Communications Society, pp. 332-336, 2004.

[3] Z. Wang and M. Zhang, "A serial concatenated scheme for LDPC code to achieve better error correction performance," in Proc. 2012 2nd International Conference on Consumer Electronics, Communications and Networks, 2012, pp. 1587-1589.

[4] T. K. Cheng, K. Sivakumar, and B. J. Belzer, Serially Concatenated IRA Codes, CoRR abs/0709.4466, 2007.

[5] Z. Wang and J. Luo, "Fountain communication using concatenated codes," IEEE Transactions on Communications, vol. 61, no. 2, pp. 443-454.

[6] G. Bonik, S. Goreinov, and N. Zamarashkin, "Construction and analysis of polar and concatenated polar codes: Practical approach," CoRR abs/1207.4343, 2012.

[7] M. Grassl, P. Shor, G. Smith et al., Generalized Concatenated Quantum Codes, 2009.

[8] S. Ding, L. J. Zhang, and H. M. Xu, "Code design and performance analysis of a novel concatenated LDPC-STBC for media communication in asynchronous cooperative MIMO systems," in Proc 7th International Conference on Wireless Communications, Networking and Mobile Computing(WiCOM), Wuhan, China, 2011, pp. 1-4.

[9] T. Muharemovic and B. Aazhang, "Information theoretic optimality of orthogonal space-time transmit schemes and concatenated code construction," in Proc. ICT, 2000, pp. 1-5.

[10] G. J. Byers and F. Takawira, "Non-binary and concatenated LDPC codes for multiple-antenna systems," in Proc. IEEE AFRICON, Gaborone, 2004, pp. 83-88.

[11] F. Simoens, H. Wymeersch, H. Bruneel et al., "Multi-dimensional mapping for bit-interleaved coded modulation with BPSK/QPSK signaling," IEEE Communications Letters, 2005, vol. 9, no. 5, pp. $453-455$.

[12] N. H. Tran and H. H. Nguyen, "A novel multi-dimensional mapping of 8-PSK for BICM-ID," IEEE Transactions on Wireless Communications, 2007, vol. 6, no. 3, pp. 1133-1142.
[13] N. H. Tran and H. H. Nguyen, "Multi-dimensional mappings of m-ary constellations for bicm-id systems," in Proc. Canadian Conference on Electrical and Computer Engineering, Saskatoon, Sack, 2005, pp. 135-138.

[14] N. H. Tran and H. H. Nguyen, "Design and performance of BICM-ID systems with hypercube constellations," IEEE Transactions on Wireless Communications, 2006, vol. 5, no. 5, pp. 1169-1179.

[15] Y. Huang, J. Lei, J. B. Wei et al., "Relay-code design for multi-user cooperation," ACTA Electronica Sinica, 2012, vol. 40, no. 10, pp. 1971-1975.

[16] N. H. Tran, T. L. Ngoc, T. Matsumoto et al., "Achieving near-capacity performance on multiple-antenna channels with a simple concatenation scheme," IEEE Transactions on Communications, 2010, vol. 58, no. 4, pp. 1048-1059.

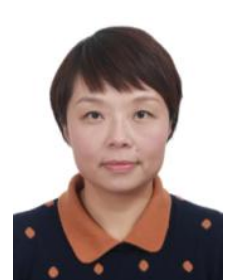

Ying Huang received the B.E. degree in electrical engineering from Xiang Tan University, Xiang Tang, China, in 2000. She then received the M.S. and Ph.D. degrees in information and communication engineering from National University of Defense Technology (NUDT), Changsha, China, in 2002, and 2014. She is currently a lecturer of the Department of Communication Engineering of NUDT. She has published in excess of 20 journal and international conference papers. Her research interests include channel codes, modulation recognition and coded cooperation.

Jing Lei received the B.E., M.S. and Ph.D. degrees in electrical engineering from the National University of Defense Technology (NUDT), Changsha, China, in 1990, 1993 and 2009 respectively. She is currently a professor of the Department of Communication Engineering of NUDT. Her research interests include channel coding technique, physical layer security technique, and cooperative communication.

Baoguo $\mathbf{L i}$ received the B.E., M.S. and Ph.D. degrees in electrical engineering from the National University of Defense Technology (NUDT) Changsha, China, in 1998, 2000 and 2004 respectively. He is currently an associate professor of the Department of Communication Engineering of NUDT. His research interests include channel coding technique, signal process etc.

Erbao Li received the B.S. degree from Northwestern Polytechnical University, Xi'an, China, in 2005, and the M.S. degree from National University of Defense Technology (NUDT), Changsha, China, in 2007, and the Ph.D. degree in communication and information from the University of Cergy Potoise, Paris, in 2012. From 2013, he is a lecturer in NUDT. His research interests include error control coding for digital communications and iterative decoding algorithms. 